
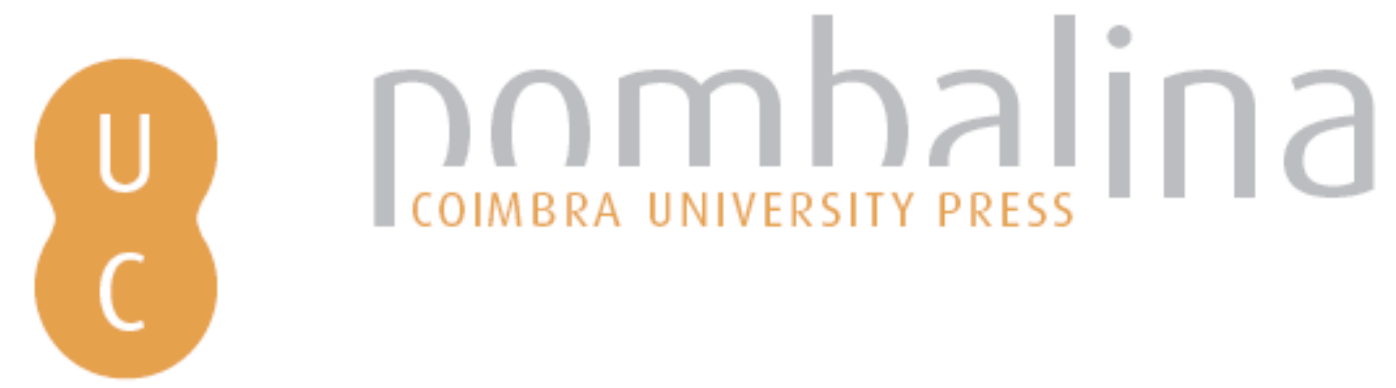

Sur deux fins malheureuses de Médée dans le théâtre français des années 1930-1940 (Henri-René Lenormand, Jean Anouilh)

Autor(es): Léonard-Roques, Véronique

Publicado por: Imprensa da Universidade de Coimbra

URL

persistente: URI:http://hdl.handle.net/10316.2/42958

DOI: $\quad$ DOI:https://doi.org/10.14195/978-989-26-1439-7_15

Accessed : $\quad$ 26-Apr-2023 16:09:33

A navegação consulta e descarregamento dos títulos inseridos nas Bibliotecas Digitais UC Digitalis, UC Pombalina e UC Impactum, pressupõem a aceitação plena e sem reservas dos Termos e Condições de Uso destas Bibliotecas Digitais, disponíveis em https://digitalis.uc.pt/pt-pt/termos.

Conforme exposto nos referidos Termos e Condições de Uso, o descarregamento de títulos de acesso restrito requer uma licença válida de autorização devendo o utilizador aceder ao(s) documento(s) a partir de um endereço de IP da instituição detentora da supramencionada licença.

Ao utilizador é apenas permitido o descarregamento para uso pessoal, pelo que o emprego do(s) título(s) descarregado(s) para outro fim, designadamente comercial, carece de autorização do respetivo autor ou editor da obra.

Na medida em que todas as obras da UC Digitalis se encontram protegidas pelo Código do Direito de Autor e Direitos Conexos e demais legislação aplicável, toda a cópia, parcial ou total, deste documento, nos casos em que é legalmente admitida, deverá conter ou fazer-se acompanhar por este aviso.

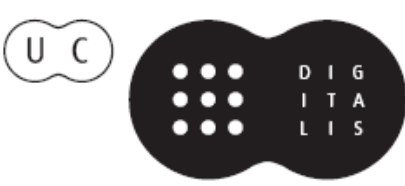




\section{Pervivencia del mundo clásico en la literatura:}

\section{tradición y relecturas}

\section{Aldo Rubén Pricco, Stella Maris Moro (coords.)}

IMPRENSA DA UNIVERSIDADE DE COIMBRA 


\title{
Sur deuX fins malheureuses de MédéE dans le théâtre FRANÇAIS DES ANNÉES 1930-1940 (Henri-René Lenormand, Jean Anouilh)
} (About the two unhappy ends of Medea in French theatre, 1930-1940 (Henri-René Lenormand, Jean Anouilh))

\author{
VÉRONIQUe LÉONARD-Roques (veronique.leonard@gmail.com) \\ Université de Bretagne Occidentale (Brest)
}

Resumé - Dans le mythe de Médée, le dénouement de l'épisode corinthien — qui se solde par une série de crimes (dont l'infanticide) - est éminemment tragique. Mais il consacre généralement la victoire de Médée sur un Jason effondré, auquel est réservée une fin misérable. Or loin d'être punie, l'héroïne parvient à s'échapper sur un mode épiphanique (manifeste dans les tragédies antiques d'Euripide et de Sénèque) qui rappelle ses origines divines. Dans le théâtre français du $\mathrm{XX}^{\mathrm{c}}$ siècle, le triomphe de Médée peut subsister. Pourtant, dans la réception du mythe, une inflexion notable s'observe dans les années 1930-1940 avec la mise en scène du suicide de la protagoniste. On examinera les modalités, les enjeux et les significations possibles de cette élimination de Médée dans les pièces d'Henri-René Lenormand (Asie, 1931) et de Jean Anouilh (Médée, 1946), non sans les rapporter à un certain affaiblissement de la figure dans la littérature européenne du XXe siècle. Chez les deux dramaturges, la réécriture du mythe porte l'empreinte des événements historiques et des tensions idéologiques dans une perspective conservatrice (opposition aux mouvements de décolonisation, résistance aux courants féministes). L'élimination de Médée, qui reste exceptionnelle dans les versions antérieures et postérieures du mythe, opère ici comme une simplification appauvrissante du scénario antique : faisant violence au programme mythologique de la figure, ce choix éradique tout ce que l'Autre conserve d'irréductible dans sa défaite même.

Mots-cLÉ: Médée, suicide, (anti)colonialisme, (anti)féminisme, appauvrissement.

Aвstract - In Medea's myth, the corintio episode's outcome - which ends in series of crimes (infantice, among them) - it's eminently tragic. But it, generally, consecrates Medea's victory over a defeated Jason, who's got an unfortunate end reserved for him. In that way, far from being punished, the heroine ends up escaping in a way similar to an epiphany (according to the ancient tragedies of Eurípides and Séneca) that reminds us her divine origins. In the twentieth century's French theater, Medea's triumph is able to survive. However, in the 1930 - 1940's decades, a remarkable inflection is observed in the myth's reception whit the staging of the protagonist's suicide. We will examine the modalities, the challenges and the possible meanings of Medea's elimination from the Henri-René Lenormand's play (Asie, 1931) and the Jean Anouilh's rendition (Médée, 1946), without overlooking this figure's undermining in the twentieth century's European literature. For the playwrights, the myth's rewriting carries the footprints of the historic events and the 
ideological tensions on a conservative perspective (opposition to the decolonization movements, resistance to the feminist's trends). Medea's elimination, which remains exceptional en the myth's early and later versions, works here as a impoverishing simplification of the ancient scene: it forces this figure's mythological program, because this choice abolishes everything that the Other preserves as irreducible in its own defeat.

Keywords: Medea, suicide, anti(colonialism), (anti)feminism, impoverishment.

Le mythe de Médée comporte un certain nombre d'épisodes dont le plus célèbre, parce que le plus fréquemment revisité d'Euripide à Mathieu Bénézet ${ }^{1}$, est l'épisode corinthien. Eminemment tragique, il se solde par une série de crimes commis par Médée (empoisonnement de sa rivale, la fille du roi Créon; double infanticide). Mais il consacre généralement la victoire de la protagoniste sur un Jason effondré, qui parfois même se suicide sur scène (comme chez Pierre Corneille²). Loin d'être punie, l'hérö̈ne parvient en effet à s'échapper sur un mode épiphanique (les pièces antiques ou classiques font intervenir force machines pour mettre en scène le char du Soleil, son ancêtre). Une telle invulnérabilité, si elle peut intriguer, rappelle les origines divines de Médée, possible figure de Grande Déesse guérisseuse et féconde dans les temps archaïques. Dans certaines versions du scénario mythique, une retraite glorieuse est même promise à la protagoniste en tant que reine à Athènes (où elle épouse Egée) puis en Asie, avant qu'elle ne connaisse une apothéose finale aux Champs Elysées (par son mariage avec Achille).

Dans les réécritures théâtrales françaises du $\mathrm{XX}^{\mathrm{e}}$ siècle, le triomphe de Médée peut subsister, comme on le voit chez Max Rouquette ${ }^{3}$ et à l'instar de ce qui se joue dans d'autres pièces européennes (Hans Henny Jahnn, Willy Kyrklund, Dario $\left.\mathrm{Fo}^{4}\right)$. Pourtant, dans la réception du mythe que livrent les années 1930 et 1940, une inflexion notable s'opère avec l'apparition d'avatars de la Colchidienne brisés, privés d'espoir et d'avenir. On examinera les modalités et les enjeux de ces fins malheureuses de Médée dans les pièces d'Henri-René Lenormand (Asie, 1931) et de Jean Anouilh (Médée, 1946). Afin d'éclairer les spécificités de ces actualisations, il conviendra de les considérer à l'aune du traitement du mythe dans quelques versions majeures du XX $\mathrm{X}^{\mathrm{e}}$ siècle.

${ }^{1}$ Mathieu Bénézet 2005.

2 Pierre Corneille (1635), Médée.

${ }^{3}$ Max Rouquette 2003.

${ }^{4}$ Jahnn (1925-1926), trad. Radrizzani 1998; Willy Kyrklund 1967, trad. Quéval et Bjurnström 1970; Dario Fo, Franca, Rame 1977, trad. Tasca 1986. 


\section{Deux éliminations de Médée au statut exceptionnel dans la CONVOCATION DE LA FIGURE AU XXE SIÈcLE}

Les pièces de Lenormand et d'Anouilh brossent le portrait tragique d'une Médée bien (trop) humaine. Accablée sous le poids de ses pertes, la protagoniste choisit d'attenter à sa propre vie, geste rare dans les versions des siècles précédents ${ }^{5}$ et dans les actualisations postérieures. La pièce $A$ sie de Lenormand, qui transpose le scénario mythique dans l'Indochine française de l'entre-deux-guerres, met en scène le retour en métropole d'un personnage du nom de De Mezzana, las de ses aventures coloniales comme de la princesse orientale qui a favorisé ses conquêtes. Ce nouveau Jason entend à présent épouser une "Européenne", fille de préfet, et renvoyer sa première femme dans son pays tout en conservant auprès de lui leurs enfants. Lenormand expose le drame de l'étrangère déracinée; il représente aussi la difficile intégration des enfants, métis torturés par leur double appartenance culturelle, sommés de choisir entre les valeurs paternelles et maternelles. Pour soustraire ses fils à un univers technique et utilitariste, la princesse les empoisonne avec de la confiture de mangue:

Non, ils ne deviendront pas les domestiques des monstres qui mangent l'espace! ils n'inventeront pas de machines. Ils ne tireront pas de leurs cervelles ces cauchemars de roues, de griffes et d'éclairs. Je sauverai l'âme que je leur ai donnée! La chose pure et sans poids que l'homme reçoit de l'homme, je la sortirai de l'enfer des chiffres et de la vitesse! ${ }^{6}$

Lenormand sacrifie le personnage de Médée, choix qu'il exhibe au dénouement. La princesse, en se précipitant par la fenêtre, affirme qu'emportée par des dragons elle retrouvera son royaume. Mais la didascalie précise que le corps de la protagoniste s'écrase sur le sol:

Layah la cherche des yeux, non pas sur le sol où elle vient de s'écraser, mais dans l'espace, parmi les nuages du couchant, qu'elle doit survoler, dans son attelage de feu?

Le suicide de Médée prend une autre portée chez Anouilh, en 1946. En écho à son modèle sénéquien ${ }^{8}$, l'héroïne éponyme clame dans la séquence finale

${ }^{5}$ Le suicide de Médée est rare. Précisons toutefois qu'avant les réécritures du XX $\mathrm{XX}^{\mathrm{e}}$ siècle considérées ici, la pièce Medea auf dem Kaukasos (1790) de Friedrich Maximilian Klinger en offre un exemple.

${ }^{6}$ Lenormand 1938: 120-121.

${ }^{7}$ Lenormand 1938: 147.

8 "Iam iam recepi sceptra germanum patrem, / spoliumque Colchi pecudis aurate tenent / rediere regna, rapta virginitas redit” (Sen. Med. 982-984): "Enfin, j'ai retrouvé mon sceptre, mon frère, mon père; les Colchidiens ont récupéré la toison du bélier d'or. Mon royaume m'est rendu; 
qu'elle “[a] recouvré son sceptre", que la "toison d'or est rendue à la Colchide" et qu'elle "[a] retrouvé [s]a patrie et [s]a virginité". Mais sa vengeance libératoire passe désormais par la mort volontaire: "Elle se frappe et s'écroule dans les flammes qui redoublent et enveloppent la roulotte" ${ }^{10}$. Dans cette œuvre, classée par le dramaturge lui-même dans ce qu'il nomme ses "Pièces noires", le suicide de Médée vient consacrer tant le règne de la solitude existentielle que la condamnation de la quête d'absolu. La princesse de Colchide est devenue une figure de la révolte, du refus des compromis, face à un Jason qui incarne l'adaptation au monde. Le personnage masculin se dit fatigué de l'intransigeance de Médée, de son "monde noir", de sa "rage de tout détruire", de ses déchirements perpétuels:

Mais je veux m'arrêter, moi [...] Faire sans illusions peut-être, comme ceux que nous méprisions, ce qu'ont fait mon père et le père de mon père et tous ceux qui ont accepté avant nous, de déblayer une petite place où tienne l'homme dans ce désordre et cette nuit ${ }^{11}$.

Médée et Jason figurent donc l'opposition entre l'exigence de l'idéal, qui frise le fanatisme, et un humanisme humble et lucide, qui prend la forme de la résignation stoïcienne. A ce stade de sa production dramatique, Anouilh a désormais choisi entre ces deux postures, ces deux tentations ${ }^{12}$. C'est ce qui explique cette revalorisation, plutôt rare aux siècles modernes, du personnage de Jason élevé ici au rang de bâtisseur:

je referai demain avec patience mon pauvre échafaudage d'homme sous l'œil indifférent des dieux. [...] Il faut vivre maintenant, assurer l'ordre, donner des lois à Corinthe et rebâtir sans illusions un monde à notre mesure pour y attendre de mouriri ${ }^{13}$.

Dans une œuvre caractérisée par son goût pour les combinaisons mythiques, Jason est rapproché d'Abel, tué par son frère Caïn en Gn IV, que les exégèses traditionnelles campent en figure emblématique de la justice et du bien. A Jason, Médée déclare en effet: "Race d'Abel, race des justes, race des riches, comme vous parlez tranquillement. C'est bon, n'est-ce pas, d'avoir le ciel pour soi et aussi les gendarmes. C'est bon de penser [...] comme tous ceux qui ont eu raison depuis toujours"14. En conséquence, dans ce système antithétique, Médée se pose

rendue la virginité que tu m'avais ravie».

${ }^{9}$ Anouilh 1947: 88.

${ }^{10}$ Anouilh 1947: 88.

${ }^{11}$ Anouilh 1947: 70.

${ }^{12}$ Borgal 1966.

${ }^{13}$ Anouilh 1947: 89.

${ }^{14}$ Anouilh 1947: 71-72. 
implicitement en figure caïnique de victime, de paria et de rebelle selon la lecture romantique du mythe (et plus précisément dans la perspective baudelairienne ${ }^{15}$ développée après l'échec de la révolution de 1848). Ce choix d'Anouilh en faveur de Jason, devenu le représentant d'une humilité et d'un relativisme valorisés a pour corollaire l'élimination du personnage de Médée, destiné au suicide au nom de la préservation de son désir d'absolu. Loin de fonctionner comme un coup de théâtre, le geste de l'héroïne est savamment préparé, la protagoniste réclamant plusieurs fois la mort au cours de la pièce ${ }^{16}$.

Ces deux actualisations théâtrales qui mettent en scène le suicide de Médée peuvent être lues comme les modulations extrêmes d'une défaite récurrente de la protagoniste dans ses convocations au fil du XXe siècle. C'est une Médée absente à elle-même, littéralement aliénée, qui est fréquemment représentée, en particulier chez les auteurs est-allemands. Heiner Müller fait ainsi sombrer Médée dans une folie qui la dépossède (forme très différente du furor sénéquien qui consiste en un combat acharné contre l'humanité, une ascèse sur la voie de la monstruosité héroïque $\left.{ }^{17}\right)$. Dans le triptyque du dramaturge daté de 1982, l'infanticide ne débouche pas sur une sortie hors du temps qui conduirait, comme chez le dramaturge latin, à un autre ordre (celui de l'éternité). Absente au monde, l'héroïne est désormais incapable de reconnaître Jason. A cette fin de la protagoniste répond celle de Médée Voix (Medea Stimmen, 1996), la transposition narrative et polyphonique livrée par Christa Wolf, qui se distingue des autres réécritures en ce que l'héroïne y est innocente de la mise à mort de ses fils. L'écrivaine renoue en effet avec des versions antérieures ${ }^{18}$ à celle d'Euripide qui a durablement ancré l'image d'une Médée infanticide dans les représentations collectives. Accusée d'avoir attiré une épidémie sur Corinthe, la protagoniste de Wolf est expulsée de la cité. Ses enfants n'en sont pas moins lapidés par les Corinthiens, ce double meurtre lui étant imputé pour les besoins du discours officiel:

So ist das. Darauf laüft es hinaus. Sie sorgen dafür, daß auch die Späteren mich Kindsmörderin nennen sollen.

Voilà. C'est ce qu'ils veulent. Que pour les générations futures je demeure celle qui a tué ses enfants ${ }^{19}$.

15 "Race d'Abel, dors, bois et mange; / Dieu te sourit complaisamment. / Race de Caïn, dans la fange / Rampe et meurs misérablement / [...] / Race d'Abel, vois tes semailles / Et ton bétail venir à bien; / Race de Caïn, tes entrailles: Hurlent la fin comme un vieux chien. ”, Charles Baudelaire (1961), "Abel et Caïn", Les Fleurs du mal [1857]: 115.

${ }^{16}$ Anouilh 1947: 36, 52.

${ }^{17}$ Dupont 1995.

${ }^{18}$ Chez Créophylos de Samos (VI' ${ }^{\mathrm{e}}$ s. av. J.-C.) et Karkinos de Nauplie (Ve ou IVe s. av. J.-C.). Voir Moreau 1994: 50-51.

${ }^{19}$ Christa 2003: 218. Médée. Voix, trad. Lance et Lance-Otterbein 1997: 254. 
Le récit se clôt sur l'évocation de Médée, terrée dans une caverne, proférant des imprécations à l'encontre des Corinthiens.

Pouvant prendre la forme extrême de l'élimination, les différents degrés d'affaiblissement du personnage signent également un refus de l'altérité. Médée est traitée de manière insistance comme l'étrange étrangère à laquelle est refusée l'intégration, dans une civilisation occidentale marquée par la domination masculine et patriarcale.

\section{UNE ALTÉRITÉ JUGÉE MENAÇANTE: SAUVEGARDER L'ORDRE COLONIAL ET LA HIÉR ARCHIE DES GENRES}

L'effondrement de Médée doit être appréhendé à l'aune de l'affirmation de la suprématie occidentale dans ce qui est, dès l'Antiquité, un mythe de la colonisation. Dans les temps les plus anciens, le voyage des Argonautes avait une valeur initiatique. Mais les explorations et les fondations de l'expédition se virent progressivement rattachées à l'histoire de la colonisation grecque, au point d'en devenir un des symboles. Le périple de la nef Argo en vint alors à marquer la jonction entre l'occident et l'orient ${ }^{20}$. A travers la confrontation entre Médée la Colchidienne et Jason le Thessalien, les versions antiques du scénario mythique questionnent les notions de barbarie et de civilisation en actualisant des références d'ordre culturel, politique et historique.

Cette antinomie acquiert une force nouvelle dans les réécritures du $\mathrm{XX}^{\mathrm{e}}$ siècle au prisme des ravages du colonialisme ou des persécutions voire des exterminations ethniques opérées par le biais de moyens techniques et scientifiques sans précédent. Dans ce contexte, le personnage du roi Egée valorisé par Euripide $^{21}$ a significativement disparu, ce qui traduit la fin d'une hospitalité possible, d'une retraite future pour Médée. Privée de droit de cité dans un Occident moderne et postmoderne qui refuse de lui faire une place, l'étrangère est plus ou moins radicalement privée d'avenir.

Müller se sert du mythe, qu'il transpose dans une forme éclatée, pour dire la crise de la Raison comme valeur de la modernité ainsi que pour invalider la notion de progrès de l'Histoire. Privilégiant les vaincus, "femmes de la Colchide" ("Weiber von Kolchis") ou "nègres morts" ("Die toten Neger") 22, il associe Médée aux catastrophes contemporaines auxquelles a conduit la volonté impérialiste

${ }^{20}$ Moreau 1994: 158-172.

${ }^{21}$ Dans la pièce d'Euripide, l'intervention d'Egée a d'abord une fonction dramatique: elle permet à Médée de prendre la mesure de l'importance d'une descendance du point de vue masculin et prépare l'infanticide. L'apparition du roi d'Athènes a aussi une fonction idéologique, dans un contexte de dissension entre Athènes et Corinthe sur fond de guerre du Péloponnèse: la scène dévalorise Créon, roi de Corinthe, en lui opposant une figure de dirigeant pieux et sage qui pratique le devoir sacré d'hospitalité.

${ }^{22}$ Müller [1982] 2002. 
et belliciste des nouveaux Argonautes. Le triptyque s'ouvre sur l'évocation du cadavre de Jason traînant au milieu de biens de consommation en voie de pourriture, ce qui motive le développement par bribes d'un récit des origines de la colonisation assumé dans le pan central par la voix de Médée. Pour Müller, face au capitalisme occidental, le salut ne peut venir que du Tiers-Monde. Dans la version d'Anouilh, en 1946, comment le choix de camper Médée en Bohémienne ne renverrait-il pas à une autre oppression, alors que venait d'être dévoilée l'entreprise d'extermination nazie du peuple tzigane et de sa culture? La représentation scénique de la roulotte dans laquelle l'héroïne habite (opposée à la richesse du palais corinthien dans lequel Jason vit désormais), l'énoncé des ethnotypes racistes qui la vouent à la marginalité ("ils nous ont parqués assez loin de leur village! Ils avaient peur que nous leur volions leurs poules, la nuit" ${ }^{23}$ ) ont une résonance particulière à la lumière du récent effondrement du IIIe Reich et de la révélation de certains de ses crimes.

On le voit, au XXe siècle les œuvres continuent à interroger l'incompatibilité prétendue de cultures que l'opposition structurale grec / barbare permet de déployer. Médée figure alors la démesure et la sauvagerie prêtées aux barbares par une civilisation occidentale qui considère qu'elle incarne l'ordre et la raison. De manière emblématique, le Créon d'Anouilh déclare à Médée: "Retourne vers ton Caucase, trouve un homme parmi ta race, un barbare comme toi; et laisse-nous sous ce ciel de raison, au bord de cette mer égale qui n'a que faire de ta passion désordonnée et de tes cris" ${ }^{24}$. Face à la figure de "femme racialisée" ${ }^{25}$ incarnée par Médée, Créon vaut comme représentant de l'ordre colonial (lequel est une composante de l'ordre patriarcal). Avec le suicide de l'héroïne, le dénouement de la pièce d'Anouilh, comme celui du drame de Lenormand, entérine le maintien d'un tel système. Asie développe aussi des arguments racistes, placés non seulement dans la bouche de Créon mais aussi dans celle de De Mezzana, l'avatar de Jason qui déclare au sujet de sa femme et leurs enfants:

Ils ne seront mes fils que quand je les aurai séparés d'elle et repeints à la couleur de ma race. Une bonne couche de rose et de blanc, voilà ce dont ils ont besoin pour m'appartenir tout à fait... Tant qu'elle vivra près d'eux ils seront exposés à l'haleine des jungles... ${ }^{26}$

Partisan de l'influence des milieux et des climats ${ }^{27}$, Lenormand revisite le mythe à des fins de psychologie géopolitique sur lesquelles on peut émettre de

\footnotetext{
${ }^{23}$ Anouilh 1947: 13.

${ }^{24}$ Anouilh 1947: 40.

${ }^{25}$ Dorlin 2008.

${ }^{26}$ Lenormand 1938: 41.

27 Blanchart 1947.
} 
grandes réserves. Car chez le dramaturge, qui se veut le témoin d'une décadence coloniale, la tragique histoire de Jason et Médée en vient à illustrer les méfaits de la transgression de ce qui serait une "loi de l'espèce"28: la nécessité de la séparation des "races". Cette pièce est aux antipodes de celle, légèrement antérieure, de l'expressionniste allemand Hans Henny Jahnn pour lequel la régénération d'une Europe en déclin ne peut provenir que du métissage et qui, pour cette raison, en vient à diviniser les fils morts de Jason et de Médée.

La pièce de Lenormand montre également qu'il n'est aucune place possible pour le merveilleux, les croyances légendaires. Ce refus de tout ce qui ne relève pas des domaines techniques et utilitaristes, rejet qui accule Médée au suicide, dit bien la "catastrophe spirituelle" ${ }^{29}$ de l'Occident dénoncée par Pasolini. Les Médée en présence sont dépourvues d'attributions magiques, dans des pièces où le spectaculaire ne sert qu'à porter sur scène leur élimination. Ainsi, lorsqu'on lui conseille de faire appel à ses pouvoirs, la princesse indochinoise de Lenormand répond qu'ils ont disparu avec la distance qui la sépare de son pays $^{30}$. Chez Wolf, Médée n'est pas une magicienne, mais une guérisseuse qui connaît parfaitement plantes et remèdes, à laquelle Jason ou les Corinthiens prêtent des pouvoirs surnaturels. Mais cette rationalisation de la figure mythique n'est pas seulement l'illustration d'un désenchantement du monde moderne ou contemporain: il permet aussi de mettre à nu les mécanismes intrinsèques du fonctionnement mythique - donc symbolique - (le travail des oppositions structurales) en dégageant ce qui y opère en termes de construction et d'expression de l'altérité (en particulier féminine). C'est plus précisément par la représentation de la défaite de Médée que sont exhibés des procédés qui contribuent à l'exclusion du féminin dans un système de domination masculine.

Médée la barbare est associée à un pôle archaïque, à la peur masculine d'un régime matriarcal ${ }^{31}$ antérieur aux temps historiques dont elle serait la dépositaire et qui menacerait le système patriarcal grec (où les femmes sont cantonnées à la sphère du domestique et exclues de l'espace civique comme des champs sociaux à forte valeur ajoutée). La réécriture féministe de Wolf montre comment, objet de calomnies, Médée est transformée en bouc émissaire, c'est-à-dire chassée de Corinthe et vouée au désespoir de la marginalisation. Dans une réflexion métapoétique, l'auteure entend déconstruire l'image négative qui est prêtée à la figure mythique depuis Euripide par des auteurs qui, très longtemps, furent

${ }^{28}$ Lenormand 1938: 84. Voir aussi 74: "L'homme, même arraché, transplanté, soufflé d'un continent à l'autre, sera toujours à sa place entre les bras d'une femme de sa couleur...”.

${ }^{29}$ Formule de P. P. Pasolini citée par Michèle Dancourt 1999: 136. On se reportera aussi au film Medea (1969) de Pasolini et à son scénario Médée, trad. Mileschi, C. (2002). Paris: Arléa.

30 "Ces paroles-là... perdent leur pouvoir... avec la distance": Lenormand 1938: 60.

${ }^{31}$ Rappelons que la thèse du matriarcat développée par J. J. Bachofen (Das Mutterrecht, 1861) est dépourvue de fondements scientifiques et véhicule une vision conservatrice en termes de catégories de genre. Voir Georgoudi 1991. 
des hommes. Elle questionne ainsi la portée idéologique de l'argument de la passion amoureuse si fréquemment avancé pour justifier les crimes de Médée, en laissant entendre qu'une telle motivation relève de représentations genrées de nature misogyne. Chez Wolf, l'héroïne ne quitte pas la Colchide sous l'effet de l'amour qu'elle éprouve pour Jason, mais parce que son pays, passant du matriarcat au patriarcat, est en proie à des changements structurels. En outre, à aucun moment la protagoniste ne tue par vengeance de femme abandonnée. On est loin de la représentation pour le moins conservatrice que livre Anouilh d'une Médée aliénée à Jason, prête à tous les crimes pour lui, "femme attachée à l'odeur d'un homme, chienne couchée qui attend" ${ }^{2}$. Une telle actualisation reproduit une vision hiérarchique des genres qui puise explicitement sa source biblique dans la création d'Eve à partir d'Adam (Gn II, 21-22), non sans renvoyer aux théories freudiennes. S'adressant à son ancêtre le Soleil, l'héroïne d'Anouilh s'exclame en effet: "Pourquoi m'as-tu faite fille? [...] Pourquoi cette plaie ouverte au milieu de moi? [...] Femme! Chienne! Chair faite d'un peu de boue et d'une côte d'homme! Morceau d'homme! Putain!"33. Ancrée dans les assignations de genre, une telle expression de la passion féminine nest pas sans pouvoir fonctionner comme un instrument de domination masculine. Les accents réactionnaires de cette représentation du féminin n'en sont que plus frappants quand l'on précise que la pièce d'Anouilh, sans avoir été remaniée, fut représentée pour la première fois en 1952, trois ans après la parution du Deuxième sexe de Simone de Beauvoir.

Les fins malheureuses de Médée exposées par Lenormand et Anouilh reflètent certaines réalités historiques et options idéologiques des années 19301940, période des plus noires marquée par l'affirmation accrue d'une hiérarchie raciale qui allait plonger le cœur de l'Europe et le monde dans la catastrophe (Auschwitz et Hiroshima en sont les symboles). Lenormand inféode sa reprise du mythe à son opposition aux mouvements d'indépendance qui, par leur amplification, conduisent à la fin de l'empire colonial français. Peu après la Libération, Anouilh exprime pour sa part la crise de l'humanisme que les exterminations ethniques de la Seconde Guerre mondiale ont provoquée tout en manifestant, par le biais de la disparition de Créon, sa perte de foi dans le politique (le dramaturge, dont l'Antigone avait été reçue en 1944 comme une célébration des faits de résistance pendant l'Occupation, est désormais accusé de collaborationnisme pour avoir pris la défense de Robert Brasillach en critiquant l'épuration ${ }^{34}$ ).

\footnotetext{
32 Anouilh 1947: 21.
}

33 Anouilh 1947: 22-23.

${ }^{34}$ Anouilh n'a pas clarifié ses positions politiques. L'interprétation d'Antigone ne cesse de faire débat, même si la majorité des critiques estiment que la pièce expose les drames de la 
Mais sa vision conservatrice du masculin et du féminin, déjà prégnante dans sa production antérieure à travers les stéréotypes et les assignations de genre (que manifeste sa réécriture du mythe d'Antigone, par exemple) semble ici plus marquée. Ces deux versions du mythe de Médée traduisent donc chez leurs auteurs de profondes résistances, aux mouvements de décolonisation pour le premier, aux courants féministes pour le second. Elles illustrent combien, au milieu du XXe siècle, le traitement du mythe porte vivement l'empreinte des événements historiques et des tensions idéologiques. Les versions majeures que livrent ensuite Heiner Müller et Christa Wolf l'attestent, dans lesquelles - en contexte postmoderne - la crise de la Raison se fait plus vivement entendre.

Chez les deux écrivains est-allemands, Médée sombre dans la folie. Mais, plus radical, le suicide sur scène que réservent Lenormand et Anouilh à l'héroïne défaite peut sembler réduire les ambiguités du mythe antique. Car, face à la question énigmatique voire scandaleuse de la femme infanticide à laquelle le matériau mythique offre une (ou des) réponse(s) symbolique(s), la tradition antique et sa réception maintiennent, comme en tension, une ouverture avec la fuite glorieuse de la coupable. En sacrifiant leur héroïne, en la supprimant, Anouilh et Lenormand opèrent sur le scénario antique une simplification appauvrissante. Le traitement de la figure de l'Autre inscrit au cœur du mythe de Médée s'en trouve affecté. Certes, dans la plupart des réécritures modernes et postmodernes (chez Jahnn, comme chez Kyrklund, Fo, Müller, Wolf ou Rouquette), Médée est bien l'Autre sur le plan ethnique et sociopolitique, cet Autre jugé inférieur que dans sa supposée mission civilisatrice l'Occident s'arroge le droit de dominer, un Autre qui apparaît aussi mystérieux et redoutable au regard des stéréotypes et assignations de genre car susceptible de menacer les positions androcentriques et les systèmes patriarcaux. Mais cette figure d'altérité, même si ses pouvoirs sont entamés, est rarement sacrifiée au point d'être mise à mort. En optant pour l'élimination de Médée, choix qui fait violence au programme mythologique de la figure, Lenormand et Anouilh éradiquent tout ce que l'Autre conserve d'irréductible dans sa défaite même.

collaboration plus que l'apologie de la Résistance. Il est certain qu'Anouilh, qui s'est élevé contre les excès de l'épuration, a été meurtri par certaines accusations de collaborationnisme portées à son encontre. Sa méfiance à l'égard du politique s'en trouve accrue, comme le montre Pauvre Bitos ou le Dîner des têtes (1956). 


\section{Bibliographie}

Anouilh, J. ([1946] 1947), Médée. Paris: La Table Ronde.

Baudelaire, C. (1961), "Les Fleurs du mal [1857]", in Euvres complètes. Paris: Gallimard, "Pléiade".

Bénézet, M. (2005), Médéa. Paris: Flammarion.

Blanchart, P. (1947), Le Théâtre de Henri-René Lenormand. Apocalypse d'une société. Paris: Société Générale d'Editions.

Borgal, C. (1966), Anouilh. La peine de vivre. Paris: Editions du Centurion.

Dancourt, M. (1999), "Le secret de Médée", in Lecercle, F. (ed.), Visible / invisible au théâtre, Textuel 36: 127-140.

Dorlin, E. (2008), Sexe, genre et sexualités. Paris: PUF.

Dupont, F. (1995), Les Monstres de Sénèque. Paris: Belin.

Fo, D., Rame, F. (1977), "Médée”, Récits de femmes et autres histoires, t. 4, trad. Tasca, V. (1986). Paris: Dramaturgie.

Georgoudi, S. (1991), "Bachofen, le matriarcat et le monde antique", in Histoire des femmes en Occident, t.1. Paris: Perrin.

Jahnn, H. H. (1925-1926), Médée, trad. Radrizzani, H. et R. (1998). Paris: José Corti.

Kyrklund, W. (1967), Médée l'étrangère, trad. Quéval, J. et Bjurnström, C. G. (1970). Paris: Gallimard.

Lenormand, H.-R. ([1931] 1938), Asie, Théâtre complet. T. 9. Paris: Albin Michel.

Moreau, A. (1994), Le Mythe de Jason et Médée. Le va-nu-pied et la sorcière. Paris: Les Belles Lettres.

Müller, H. ([1982] 2002), "Verkommenes Ufer Medeamaterial Landschaft mit Argonauten", in Werke 5, Frankfurt, Suhrkamp. "Rivage à l'abandon Matériau-Médée Paysage avec Argonautes", trad. Jourdheuil, J. et Schwarzinger, H. (1985), Germania. Mort à Berlin. Paris: Minuit.

Rouquette, M. (2003), Médée [1989 pour la version occitane, 1992 pour la traduction française]. Montpellier: Espaces 34.

Sénèque, Medea, in Tragoediae (1966). Bologna: Editrice Composituri.

Wolf, Ch. ([1996] 2003), Medea Stimmen. München: Deutscher Taschenbuch Verlag. Médée. Voix, trad. Lance, A. et Lance-Otterbein (1997), R. Paris: Fayard. 